\title{
What Details are Needed for Wireless Simulations? - A Study of a Site-Specific Indoor Wireless Model
}

\author{
Mustafa Al-Bado Cigdem Sengul Ruben Merz \\ Telekom Innovation Laboratories at TU-Berlin \\ \{mustafa, cigdem\}@net.t-labs.tu-berlin.de, \{ruben.merz\}@telekom.de
}

\begin{abstract}
-
The wireless networking community continuously questions the accuracy and validity of simulation-based performance evaluations. The main reason is the lack of widely-accepted models that represent the real wireless characteristics, especially at the physical (PHY) layer. Hence, the trend in wireless networking is to rely more and more on testbeds, which on one hand bring more realism to network and protocol evaluation, but on the other hand present a high implementation barrier before an idea is ready to be tested. Therefore, realistic network simulators are still very much needed to reduce the time and effort for "concept testing" of novel ideas. In this case, the main question is how detailed should wireless simulators be to evaluate network and protocol performance. In this paper, we attempt a first answer to this question by using the Berlin Open Wireless Lab (BOWL) indoor model (BIM) in the ns-3 simulator. BIM includes several measurement-based models to characterize wireless communication such as frame detection ratio (FDR), frame error ratio (FER), capture and interference models. Through extensive measurements, we analyze the accuracy that we obtain with these PHY-layer models. Our experiments also show whether the detailed models at the PHY layer play an important role to represent transport layer performance in simulations.
\end{abstract}

\section{INTRODUCTION}

The use of simulations for performance evaluation, in particular packet-level simulations, is today considered a big concern due to the lack of accuracy in wireless models, especially at the physical layer (PHY) [1]. As a result, wireless network testbed deployments have become increasingly important for the development and evaluation of network protocols and algorithms. However, as testbeds require a high investment in time, effort and resources, addressing the challenge of designing more realistic PHY models for simulation once again becomes important.

Certainly, modeling the wireless PHY layer is a gargantuan task due to (1) the number of factors that need to be considered (e.g., adjacent channel interference [2] or frame error rate with overlapping packet transmissions) and (2) the time scale difference between events at the PHY layer and the MAC layer and above [3], [4]. In this paper, we focus mainly on radio wave propagation, packet capture and interference in wireless simulators. Simulators use different propagation models to assign a received signal strength (RSS) at any potential receiver node for every transmitted packet. Several works [5], [6], [7], [3], [8] illustrated how different propagation models do indeed add to the inaccuracy of wireless network simulation. In previous work, we underlined the importance of measurementbased site-specific link models [8] to be able to represent different characteristics of a wireless network. In this paper, our objective is to determine the necessary and sufficient detail for our wireless models to create a realistic representation of our BOWL indoor wireless network testbed [9]. Our overarching goal is to incite the creation of a collection of site-specific models in simulators in the community, which will lead to evaluation of new ideas in simulators in as many diverse conditions as possible.

In [8], we developed the BOWL Indoor Model (BIM), a measurement-based site-specific link model of our testbed for ns-3, a well-known packet-level network simulator [10]. The first version of BIM included propagation, frame detection and frame error ratio models. The frame detection ratio (FDR) model represents the detection of a frame on the medium and the acquisition of the timing of the frame. The frame error model (FER) handles the events after detection, which lead to handing the frame over to the MAC layer if the payload is successfully recovered, and otherwise dropping a frame. In this work, we extend the FER model to provide more linklevel accuracy, and also add a capture and interference model. The main goal of our work is to understand how much detail BIM should include to represent the upper layer performance of BOWL in a simulator. We summarize our contributions as:

- A comparison of different frame error ratio (FER) models of increasing precision at the PHY layer. Our comparison is performed against extensive measurements in an IEEE 802.11a wireless network testbed.

- Incorporation of capture and measurement-based interference models into BIM to evaluate performance with multiple concurrent senders.

- Investigation of whether precision at the PHY layer impacts performance evaluations at the transport layer.

Our results indicate that links in our wireless testbed show high variability (e.g., highly varying FER and RSS ranges) and can be roughly categorized as: (1) links that can be modeled with less detail (i.e., simple-model links) and (2) links that need to be modeled with more detail (i.e., complexmodel links). Hence, knowing which links fall under which category helps reduce the measurement effort and modeling complexity required to represent a wireless network in a simulator. Second, even for the complex-model links, we show 
that high precision in FER does not necessarily provide more accurate results at the transport layer. On the contrary, FDR, capture and interference models play a significant role.

The rest of the paper is outlined as follows. In Section II, we review the background and the related work. In Section III, we describe our testbed and our BOWL indoor model. Section IV describes our measurement and simulation setup for the enhanced BOWL indoor model, which includes extended FER models, capture and interference models. In this section, we also present the results from our measurement study of the impact of FER accuracy and present PHY-layer results for the capture and interference models. In Section V, we evaluate the higher layer performance with different propagation models in the simulator and the performance observed during measurements. Finally, we conclude in Section VI.

\section{BACKGROUND \& RELATED WORK}

There are a number of network simulators commonly used by the wireless community, such as ns-2 [11], ns-3 [10], Opnet [12] and QualNet [13]. The numerous wireless communication models in these simulators is a sign of the challenge in designing realistic models. In this section, we will first review the existing models, focusing especially on ns-3 [10], which is the simulator used in this paper. We chose to work with ns-3 mainly for its emphasis on integration with real systems. Finally, we conclude this section with a short summary of the related works on improving wireless simulation accuracy.

\section{A. PHY/MAC Layer Models in Current Simulators}

Most current simulators implement models for a wireless network interface controller based on the IEEE 802.11 PHY/MAC standard. All simulators include the typical stochastic channel propagation models: the Friis model, the two-ray ground model and a shadowing model [14], [15], [16], [11]. In terms of interference modeling, ns-2 relies on a threshold-based model where the signal strength is compared against a single node at a time. Several works cautioned against the realism of this model, and proposed the use of a cumulative interference models that takes into account all interfering nodes [17], [18], [19]

In the simulator of our choice, ns-3, the PHY layer is implemented by the YansWifiPhy class (see [20], [21]). In terms of propagation models, ns-3, contains various additional models such as two log-distance models (LogDist and ThreeLogDist) applicable to indoor propagation modeling, a constant loss model, the COST-Hata model for urban-area propagation (see Chapter 4 from [22]), a fast-fading Nakagami model for modeling multi-path effects [16] (which can also be used for Rayleigh fading), and the so-called Jakes propagation model for Rayleigh fading in mobile environments [23].

In ns-3, the PHY layer can be in four states: TX, SYNC, CCA_BUSY and IDLE. In the TX state, the PHY is currently transmitting, and in the SYNC state, the PHY is synchronized on a signal to receive the associated frame. The CCA_BUSY represents the energy detection by the wireless radio. If the PHY is not in any of these states, it is IDLE. The energy of the received signal is determined by the chosen propagation model. When the last bit of the synchronized packet $k$ is received, a packet error probability, $P_{e r r}(k)$, is calculated and used to decide whether the packet $k$ is successfully received or not. To make this decision, a uniformly random number is drawn and compared with $P_{e r r}(k)$. The value of $P_{e r r}(k)$ is computed based on the upper-bound of the error that may be present on the part of the packet received at a given interval $l, P_{e}(k, l)$. This allows representing the changes in error probability when different parts of the packet are subject to different levels of interference. More specifically, $P_{\text {err }}(k)$ is computed as [20]:

$$
P_{e r r}(k)=1-\prod_{l}\left(1-P_{e}(k, l)\right) .
$$

Here, $P_{e}(k, l)$ is derived based on an interval $l$ of time $t$, where the bit error rate, $B E R(k, t)$ and transmit rate, $R_{b}(k, t)$ are constant. The calculation of $P_{e}(k, l)$ also takes into account forward error correction in IEEE 802.11a. $B E R(k, t)$ is a function of the signal to noise and interference ratio, $\operatorname{SINR}(k, t)$, based on the corresponding equations for different modulation schemes (see Equations (5)-(8) in [20]). $\operatorname{SINR}(k, t)$ is calculated as [20]:

$$
\operatorname{SINR}(k, t)=\frac{S_{k}(t)}{\sum_{m \neq k} S(m, t)+N_{f}},
$$

where $\sum_{m \neq k} S(m, t)$ is the interference noise from other packets $m$ and $N_{f}$ is the noise floor, which is a characteristic of the receiver circuitry.

While ns-3 implements a quite complex model to represent interference from concurrent senders, it currently does not take into account the capture effect. With the capture effect, a stronger frame that arrives during the reception of a weaker frame can still be received successfully. Based on different cards, different capture effects can be modeled. For instance, with Prism chipsets a stronger frame can only be captured if it arrives within the weaker frame's preamble time [24]. On the other hand, Atheros chipsets implement MIM (Message in Message), which allows capturing frames even if they arrive after the preamble time of the first frame [25], [24], [19]. In Section IV, we describe the measurement-based interference model and the capture model we adopted in further detail.

\section{B. Improving Simulation Accuracy through Measurements}

Even though PHY layer models become more sophisticated, several measurement studies show that the existing models, typically the channel propagation models, are not able to represent real environments accurately. This inaccuracy has farreaching effects [5], [6], [7], [8]. For instance, due to assuming more wireless links are present than reality, routing layer performance might be overestimated [26]. More importantly, the comparison of two routing protocols may yield completely different results (i.e., $x$ outperforms $y$ in the simulator but not in reality). Nevertheless, wireless networking research still needs simulation to be able to evaluate protocols independently of the real system complexity and also to understand the 

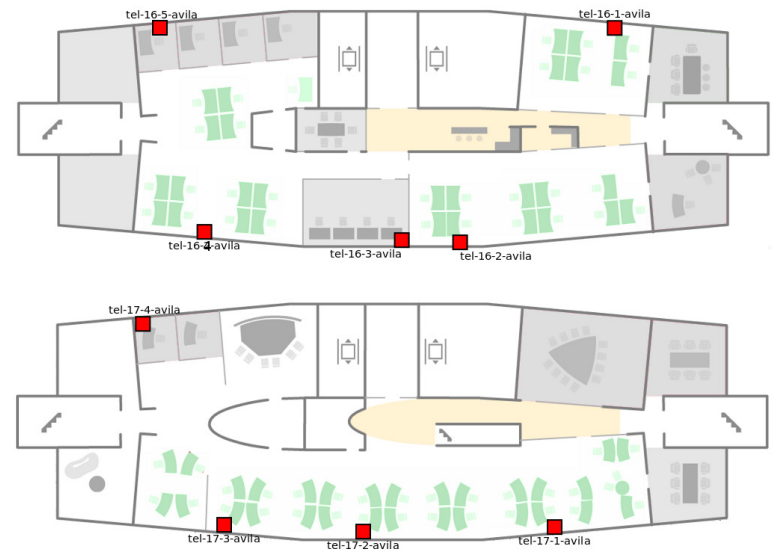

Fig. 1. The BOWL indoor testbed spans two floors. There are five nodes on the 16th floor (top picture) and four nodes on the 17th floor (bottom picture). The host names are indicated with the convention: tel-floor-node.

performance in different network topologies, which is not possible to test in a single testbed.

Hence, there are several works proposed to improve accuracy, such as calibrating existing models to fit the real network behavior by using testbed measurement results. In [7], the results show that calibrating the ns-2 shadowing model helps to match simulation results with the measurements in terms of packet delivery ratio and network topology. In [3], two different measurement methods were used for calibration. First, the RSS between each pair of nodes in the testbed was measured. Second, two nodes were moved to different locations in the building, and the RSS between these two nodes was recorded. Using these measurements, the authors model propagation combining path-loss and log-normal shadowing and also model the deferral probability between two nodes. Their results based on ns- 2 show that measurementbased models are able to achieve higher accuracy, whereas uncalibrated models either underestimate or overestimate the capacity. However, these studies do not take into account the effect of radio-specific properties, such as preamble detection, capture [24], adaptive noise immunity or weak signal detection [27], [28]. These issues play an important role as shown in [8], [5], [6], [24]. Hence, while simulation models do benefit from measurements, measuring the entire range of different factors and calibrating a single model based on these measurements are significant challenges. Therefore, in this paper, we present a site-specific model, which allows predicting channel characteristics for a link and evaluate what details are needed to represent the characteristics of our links in our indoor BOWL testbed.

\section{BOWL Testbed AND Indoor Simulation Model}

Our simulation models are based on measurements carried out in the BOWL indoor testbed [9] at Telekom Innovation Laboratories, in Berlin, Germany. In this section, we first describe our testbed and then, explain our BOWL Indoor Model (BIM), which forms the basis for this work.

\section{A. System Description}

The BOWL indoor testbed consists currently of nine nodes, five of them are deployed in one floor, and the remaining four reside on the floor above (see Fig. 1). The host names indicate the floor and the node numbers. For instance, tel-16-2 is the second node on the 16th floor. Each node has a Gatework Avila GW2348-4 platform with 64 Mbyte of RAM, an Intel XScale IXP425 $533 \mathrm{MHz}$ processor (ARM architecture) and two Wistron CM9 miniPCI IEEE 802.11abg wireless network interface cards (NIC), and a $8 \mathrm{dBi}$ gain omnidirectional antenna (with a $2 \mathrm{~dB}$ loss because of cabling). The wireless NIC is an Atheros AR5213A [29]. We use OpenWrt 8.09.2 with Linux kernel 2.6.26.8 as the operating system. The wireless driver is the version maintained by OpenWrt, with revision number 3314 with HAL 20090508. Additionally, all nodes have a dedicated Ethernet management interface of $100 \mathrm{Mbit} / \mathrm{s}$ capacity, which is used to collect measurement results on a central server. To generate experiment traffic, we use one dedicated load generator machine (loadgen) with Intel(R) $2.80 \mathrm{GHz}$ processor, four $\mathrm{CPU}$ cores and $6 \mathrm{~GB}$ RAM. The operating system is Linux version 2.6.32.

\section{B. BOWL Indoor Model (BIM)}

Our main simulation model, the BOWL indoor model (BIM) [30], was originally proposed in [8]. It is a measurement-based model of the BOWL indoor network and comprised a radio propagation model, a frame detection ratio (FDR) model and a frame error ratio (FER) model. FDR is the ratio of all the detected frames (i.e., includes the frames with errors) to the transmitted packets. FER is the ratio of frames with errors to FDR. A frame has an error when it does not pass the Cyclic Redundancy Check (CRC).

BIM was added to ns-3 as a new propagation model. It made the following modifications to the YansWifiPhy class:

- Support for feeding measurement-based RSS distributions

- Per-rate FDR threshold

- A FER model with RSS and modulation as parameters

- Transmit power behavior of Atheros hardware

- Recording of dropped frames in the radiotap trace

Our model is based on per-link RSS distributions. Based on our measurements, for each link and for each combination of data-rate and channel frequency, we build an empirical cumulative distribution function (ECDF) of the RSS. Our data includes only the RSS of detected frames (i.e., excludes frames that were dropped by the radio signal detection unit). As we know exactly how many frames were transmitted, for each lost frame, we represent its RSS as the corresponding FDR threshold (i.e., the noise floor plus a data-rate specific correction factor, see [8] for more details). In the simulator, the RSS value for a given frame is obtained simply by sampling the RSS distribution of the corresponding link taking into account its data-rate and channel. The frames with RSS below the FDR threshold are dropped. Frames equal to or higher than the threshold are passed to the FER model, where the RSS and data-rate of the frame is used to look up the corresponding 
TABLE I

SUMMARY OF EXPERIMENTS WITH RATE AND POWER LEVELS USED

\begin{tabular}{|l|l|}
\hline \multicolumn{2}{|c|}{$\begin{array}{c}\text { Broadcast Experiments for } \\
\text { Database Construction } \\
\text { and PHY Layer Validation }\end{array}$} \\
\hline \hline Simulation database & 6,24 and 54 Mbps. 8 power levels. \\
\hline FER validation & 6 and 54 Mbps. TX power 12 $\mathrm{dBm}$. \\
\hline Capture \& interference validation & $\begin{array}{l}6 \text { and 54 Mbps. 17 power levels } \\
\text { in total for interferer and transmitter. }\end{array}$ \\
\hline \hline \multicolumn{2}{|c|}{ Unicast Experiments for Transport Layer (TCP and UDP) Validation } \\
\hline \hline Single Flow & 6 and 54 Mbps. TX power 12 $\mathrm{dBm}$ \\
\hline Multiple Flows (2-3 Flows) & 6 and 54 Mbps. TX power $12 \mathrm{dBm}$. \\
\hline
\end{tabular}

frame error probability $p$. This probability is then compared to a uniformly sampled random variable $q(0 \leq q \leq 1)$. If $q>p$, the frame is successfully received. Else, the frame contains an error and is dropped.

\section{The ENHANCEd BOWL INDOoR Model}

In the original BIM model, in contrast to our per-link propagation and FDR models, the FER model is a networkbased model, obtained by aggregating measurement traces for all links in the network. In this paper, we extended BIM to provide different levels of precision in its FER model. Furthermore, we included two models to represent capture and interference effects. These extensions, and the new measurement experiments to create and validate them, are the topic of this section.

\section{A. Measurement and Simulation Set-up}

All our measurement study was conducted in the BOWL indoor testbed, with nodes using a single wireless interface configured for IEEE 802.11a [31]. All experiments were run on channel 44 with packet size of 1024 B (Bytes). Before each experiment, we always checked that the channel was free of other interfering transmitters. Based on experiments with different packet sizes (64 B and $512 \mathrm{~B}$ ), we have noted that for a small fraction of links (3 out of 51), packet size may have an effect on observed frame errors at a given transmitter power level. An extension of the model for different packet sizes for particular links is planned but left for future work.

We ran several broadcast and unicast experiments to construct the simulation database of links, as well as for validating our results. Broadcast experiments are used to build the simulation database as well as for PHY-layer simulation validations. In broadcast experiments, senders were set up in ahdemo mode ${ }^{1}$, which disables the transmission of all management frames (e.g. beacons). This allows us to run experiments without any control overhead. The receivers were set up in monitor mode $e^{2}$, which allows us to gather the link layer information (e.g., RSS) using the so-called radiotap header ${ }^{3}$. In all broadcast experiments, there is always only one sender at a given time. In all cases, each experiment comprises several measurement runs for each sender. Because the accumulated duration of all runs for a given node can be on the order of an

\footnotetext{
${ }^{1} \mathrm{http} / / /$ madwifi-project.org/wiki/UserDocs/AhdemoInterface

${ }^{2} \mathrm{http} / / /$ madwifi-project.org/wiki/UserDocs/MonitorModeInterface

${ }^{3}$ http://madwifi-project.org/wiki/DevDocs/RadiotapHeader
}

hour or more, a given sender does not perform all the runs in a row. After each measurement run, another sender was chosen. All measurement runs for a given experiment have the same setup. Tcpdump was used to collect traces at each receiver. These traces were redirected to and stored on a central server using the Ethernet management interface. For all experiments, CBR UDP broadcast traffic is generated using the Iperf tool. Because of the broadcast transmission, no RTS/CTS control frames are transmitted. All experiments were conducted during the work days (i.e., excluding weekends). For the sake of studying the day-night effect, each experiment was repeated 20 times.

The differences among the different broadcast experiments are the number of rate and power levels used. These differences are summarized in Table I. For instance, for database construction, we use all the combination of the data-rates in IEEE 802.11a and eight different transmit powers: $0,2,4,6,8$, 10,12 and $13 \mathrm{dBm}$. Here, changing the transmit power helps to create varying RSS conditions at the receivers, which leads to building a more accurate database. For validation we pick two rates (the lowest, $6 \mathrm{Mbps}$ and the highest, $54 \mathrm{Mbps}$ ) and a single power level ( $12 \mathrm{dBm}$ was chosen as it is the highest common power for different rates for Atheros cards).

Unicast experiments are used to validate the transport layer performance based on the new BIM extensions. In these experiments, before and after each experiment, broadcast sessions were run to update the simulation database for frame detection. In unicast experiments, all nodes were setup in ahdemo mode. Each experiment used either TCP Reno or UDP. In these experiments, we switched to using ns-3 as a traffic generator to have a consistent protocol stack (e.g., the same TCP implementation) for both measurements and simulations. To this end, we used the OnOffApplication class with an always-on setting. We tried to saturate the channel for the chosen modulation and coding rate. We achieved $5 \mathrm{Mbps}$ for 6 Mbps transmit rate, but achieved $20 \mathrm{Mbps}$ for $54 \mathrm{Mbps}$ transmit rate due to CPU limitations.

This ns-3 simulator ran in loadgen connected to the BOWL indoor testbed (see Section III-A). We used ns-3 version $3.10^{4}$. The simulated topology represents our node deployment shown in Fig. 1. In simulations, the transmission of beacon management frames is also disabled. We use our BIM model with IEEE 802.11a PHY settings. We set the noise floor value to the one in our measurement traces. Each simulation scenario was run for ten times using different seeds. All simulation results are presented with their $95 \%$ confidence intervals.

\section{B. Enhanced FER Models}

Before designing more advanced FER models, as a first step, we verified whether the FER performance varies from receiver to receiver. For this evaluation, we used the "simulation database experiments" (see Table I). Based on these experiments, where each sender takes turns to transmit frames with eight different power and rate levels, we can identify the

\footnotetext{
${ }^{4}$ The latest release 3.13 does not contain any changes that affect our work.
} 


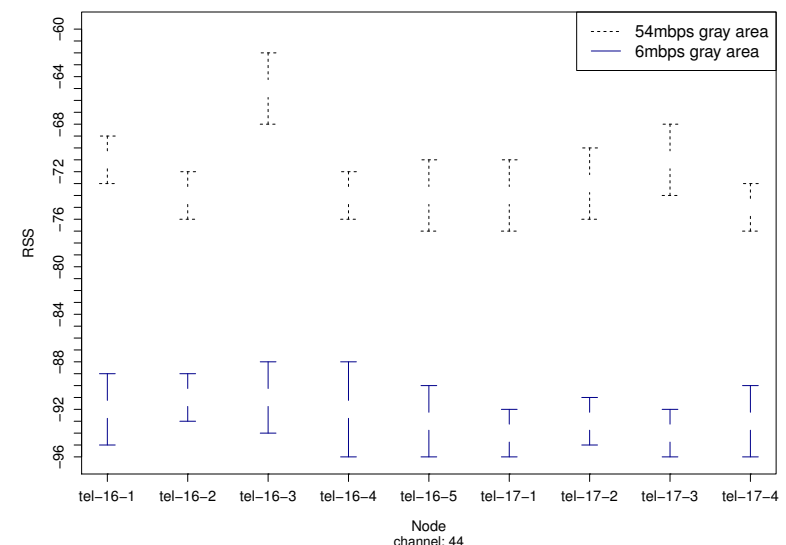

Fig. 2. The RSS range per receiver, where $5 \%<F E R<95 \%$ (gray area) for 6 and 54 Mbps. The gray area deviates per receiver per data rate.
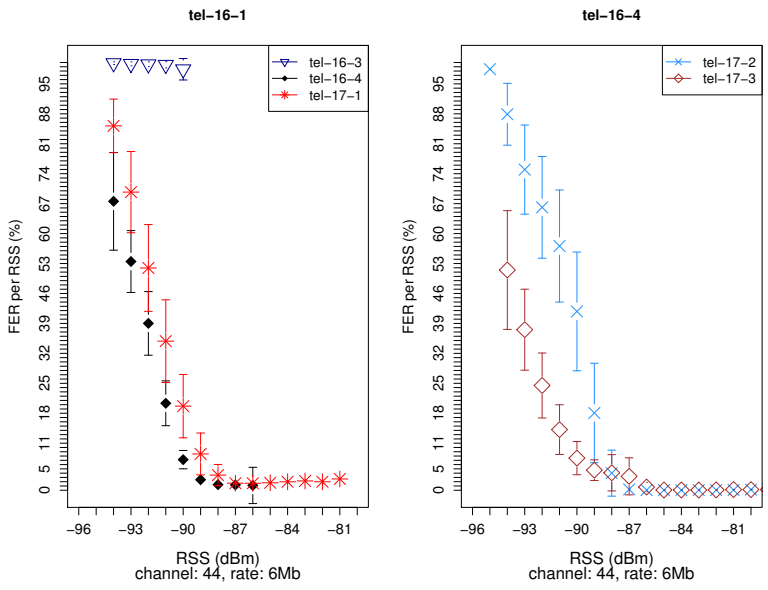

Fig. 3. The per-link FER performance for selected links for receivers tel-16-1 and tel-16-4.

range of RSS values with FER higher than $5 \%$ and lower than $95 \%$. We call this range as the "gray area", as we consider that a FER value higher than $95 \%$ correspond to significantly weak links, and a FER value lower than $5 \%$ represent strong links. Fig. 2 shows the gray area for each receiver in our testbed with two different rates: $6 \mathrm{Mbps}$ and $54 \mathrm{Mbps}$. We observe that for each rate both the width, and the RSS values covered in the gray area deviate per receiver. For instance, while the typical RSS range for receivers at $54 \mathrm{Mbps}$ lie between -76 and $-72 \mathrm{dBm}$, for te $1-16-3$ this range is between -69 and $-62 \mathrm{dBm}$. Another example is tel-16-4, where the gray area for $6 \mathrm{Mbps}$ is significantly larger than others (between -96 and $-88 \mathrm{dBm})$. We also evaluated per-link FER variation. Fig. 3 shows selected links of two receivers, tel-16-1 and tel-16-4 as examples. For tel-16-1, we see that the links from tel-16-4 and tel-17-1 improve in terms of FER as the RSS at tel-16-1 increases (i.e, as these transmitters increase their transmit powers). On the other hand, the link from tel-16-3 is a very weak link and for the cases tel-16-1 can hear this node, the FER remains $\approx 95 \%$. For tel-16-4, both transmitters, tel-17-2 and tel-17-3 experience low FER as the RSS at tel-16-4 increases. However, for both nodes, the FER performance in the gray area is significantly different, the link from tel-17-3 being stronger.

Based on receiver-based and link-based measurement results, we conclude that a more detailed FER model might achieve better accuracy in simulations. To evaluate how much benefit we can get from a more detailed model, we consider two extensions. The Receiver-based FER model assumes that receiving the packet correctly depends on the receiver's environment and hardware. Hence, each receiver has a different FER model based on the aggregated FER measurements from all senders. To construct the simulation database for this model, we used the same traces as the network-based model. However, in this case, the traces were aggregated based on the receiver node and the data-rate. When there were not enough frames received by a receiver at a particular RSS, we used the network-based model to fill this gap via interpolation. The Link-based FER model is the most detailed FER model as there exists a FER model for each sender-receiver pair. This model is especially useful when there is high link diversity in the network. To construct the link-based FER database, the measurement traces were aggregated based on a given link and data-rate. Again, we fill the gap in the link-based FER tables using receiver and network-based tables and interpolation.

To evaluate the benefit from the two new models, we compare them against (1) BIM without our FER model (i.e., ns-3 BER-based model is used to decide whether a packet is received correctly or not, see Section II-A), (2) BIM with network-based FER model, and (3) BOWL testbed measurements. Fig. 4(a) and Fig. 4(b) depict the case when the senders used 6 and $54 \mathrm{Mbps}$ transmission rates. The graphs show the frame reception ratio (FRR) for all links in ascending order. FRR is actually FDR $\times(1$-FER $)$, which is the ratio of the detected packets that passed the CRC check. We see that in both cases, that majority of the links fall either in the category of strong links ( $>95 \%$ FRR) or do not exist $(\approx<0 \%$ FRR $)$. Hence, these links can be modeled with simple models. On the other hand, for a small number of links (5 out of 51 for both 6 and $54 \mathrm{Mbps}$ ), more detailed models significantly improve the simulation performance of these links. The improvements are especially significant for $54 \mathrm{Mbps}$, as it is a less robust rate compared to $6 \mathrm{Mbps}$ and hence, modeling the frame error behavior precisely is more important. The results show that the link-based FER model can reduce the normalized root-mean square error (NRMSE) to $3.24 \%$, where as the NRMSE for receiver-based model is $7 \%$, networked-based model is $10.81 \%$, and the ns-3 BER model is $12.79 \%$. Therefore, at the PHY layer, indeed, a more detailed model helps improve representing "gray area" links. In Section V, we further evaluate how much difference detailed models make in comparison to more simpler models.

\section{Interference and Capture Models for BIM}

In our previous work [8], and the previous subsections, we only considered the cases with a single sender at a time. To 


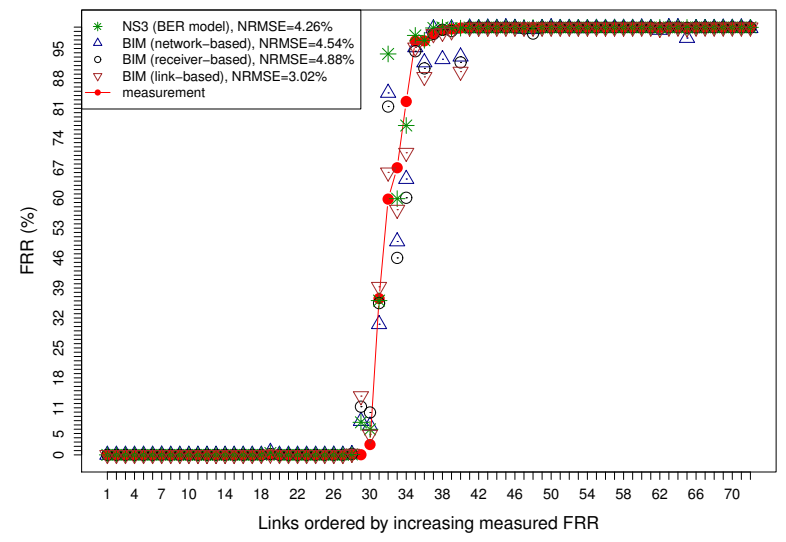

(a) 6 Mbps

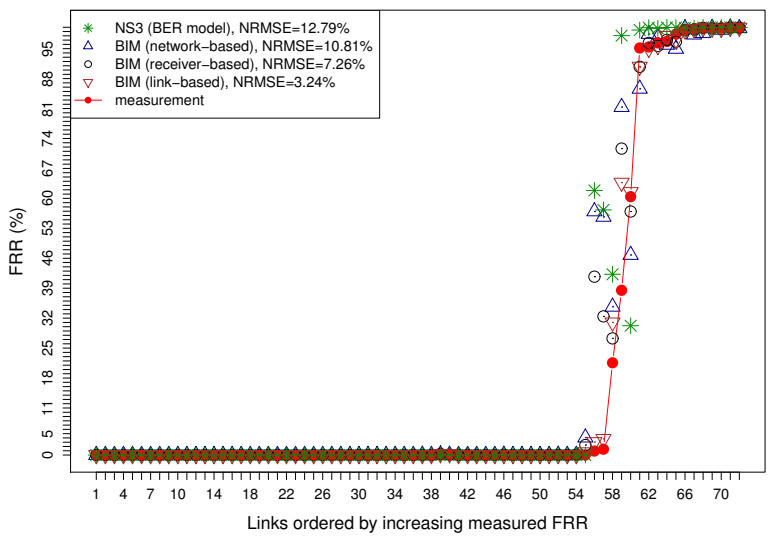

(b) $54 \mathrm{Mbps}$

Fig. 4. FER validation for two different rates. Five out of 51 links are complex-model links with $5 \%<F R R<95 \%$.

be able to represent the performance with multiple senders, we need to add an interference model to BIM. As explained in Section II-A, ns-3 has already a quite complex interference model based on BER calculations. The model calculates the SINR taking into account that multiple packets might be overlapping at different times (see Equations 1 and 2).

For BIM, we adopt a measurement-based interference model based on [32] (see Equations 2-7 in [32]). Our goal is to decide whether a receiver $r$ will correctly decode a packet from sender $s$, while there are competing packets. In [32], the probability of correctly decoding packets from $s$ to $r$ is represented as $p_{r}\left(S_{s r}\right)$, where $S_{s r}$ true incoming signal energy at the receiver $r$ from $s$. Given the average external interference, $\overline{I_{r}}$, and $\delta_{r}$, which is the SINR threshold for the receiver $r$ to be able to decode a packet for a given modulation, $p_{r}\left(S_{s r}\right)$ is calculated as:

$$
p_{r}\left(S_{s r}\right)=\operatorname{Prob}\left[\frac{S_{s r}}{I_{r}+N_{f}} \geq \delta_{r}\right]
$$

where $N_{f}$ is the noise floor. Using the RSS measurements, $S_{s r}$ can be approximated as $S_{s r} \approx \overline{R_{s r}}-\overline{I_{r}}$. Values of $\overline{I_{r}}$ are calculated based on the assumption that the variation in RSS

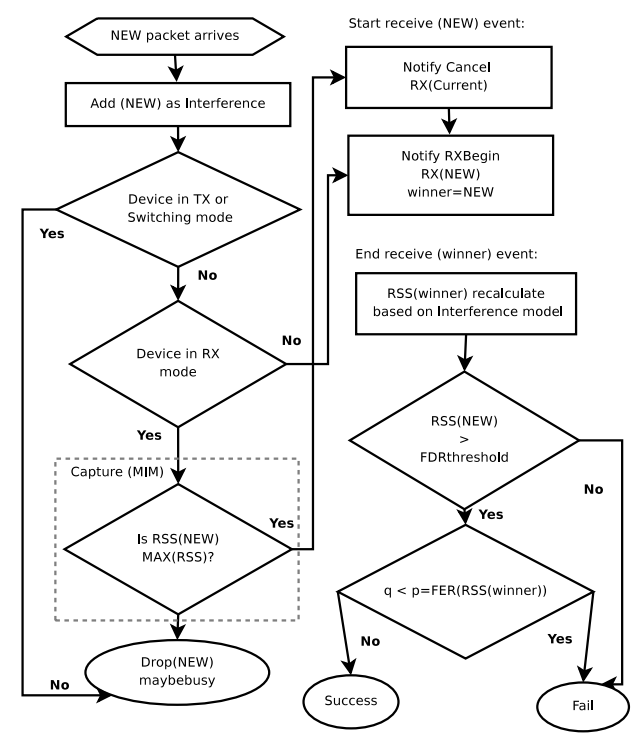

Fig. 5. The implementation of capture and interference model in BIM.

measurements stem mainly from external interference. Hence, $\overline{I_{r}}$ can be calculated as the mean excess of RSS values over the corresponding minimum measured values.

The delivery probability from $s$ to $r$, when $t$ is interfering is denoted as $p_{r}\left(S_{s r}, S_{t r}\right)$, and approximated as:

$$
\begin{aligned}
p\left(S_{s r}, S_{t r}\right) & =\operatorname{Prob}\left[\overline{\overline{R_{t r}}}-\overline{I_{r}}+\overline{I_{r}}+I_{r}+N_{f} \geq \delta_{r}\right] \\
& =\operatorname{Prob}\left[I_{r} \leq \frac{\overline{R_{s r}}-\overline{I_{r}}}{\delta_{r}}-\left(\overline{R_{t r}}-\overline{I_{r}}\right)-N_{f}\right]
\end{aligned}
$$

While based on our measurements, we have FDR and FER for each independent sender, we do not have measurements for multiple senders. Therefore, we adopt the hypothetical single sender method (HSSM) from [32]. The RSS from this hypothetical sender is $R X_{s r}^{t}$. Given that this hypothetical sender has the same interference threshold in Equation 5:

$$
\begin{aligned}
\frac{R X_{s r}^{t}-I_{r}}{\delta_{r}}-N_{f} & =\frac{\overline{R_{s r}}-\overline{I_{r}}}{\delta_{r}}-\left(\overline{R_{t r}}-\overline{I_{r}}\right)-N_{f} \\
R X_{s r}^{t} & =\overline{R_{s r}}-\delta_{r}\left(\overline{R_{t r}}-\overline{I_{r}}\right)
\end{aligned}
$$

Using $R X_{s r}^{t}$, then we can apply the FDR and FER model to decide whether an incoming packet from $s$ can be received in the presence of $t$. Note that, as also stated in [32], this model ignores the temporal considerations (i.e., whether the interfering packet starts transmissions before or after the main packet). This is, in our implementation, handled through the capture model, which represents the MIM (Message in Message) (see Section II-A) capability of our Atheros radios. Note that, by default, ns-3 assumes that the radio locks on the first packet and drops all other incoming packets (even though their interference is taken into account).

To add interference and capture models to BIM, we create a new BIMInterference class. Fig. 5 shows the flowchart of our implementation. In our implementation, when a NEW packet arrives, it is added to the "Interference" list. Then, the 
status of the radio is checked. If the radio is in TX or switching modes, the packet is dropped. If the radio is IDLE, the radio is switched to RX, and the reception of the new packet is notified. On the other hand, if the radio is already in RX mode, then it is checked whether the RSS of the NEW packet is higher than the current packet. If yes, the MIM model is applied, and the current packet is canceled and the radio starts receiving the NEW packet. If no, the NEW packet is dropped. We denote the packet that the radio decides to receive as the winner. At the end of the receive duration of the winner, the RSS value is recalculated, taking into account all the overlapping interfering packets. Next, the FDR model is used to decide whether the packet can be detected. Then, based on the FER model, we decide the fate of the winner as either Success or Fail.

In the rest of the section, we evaluate the BIM interference and capture model (i.e., HSSM and capture model). Similar to the previous evaluations, we investigate 6 and 54 Mbps datarates. The experiment includes three nodes: receiver, sender and interferer. To evaluate different interference conditions, 15 different transmit power-levels between the sender and the interferer were tested for a duration of 120 seconds each. For $6 \mathrm{Mbps}$, the difference between incoming signal powers of the transmitter and the interferer at the receiver ranges between $1-15 \mathrm{~dB}$. This range is $18-34 \mathrm{~dB}$ for 54 Mbps.

We compare the BIM model against 4 different models (see Figs. 6 and 7). The model with the least detail is the default ns-3 model described in Section II-A (referred as NS3 BER $\&$ no capture model in the figures). Each model has varying levels of detail e.g., either includes link-based FER model, uses HSSM instead of the ns-3 interference model or has the capture model. The results show that for both $6 \mathrm{Mbps}$ and $54 \mathrm{Mbps}$ data-rates, the capture model has a significant impact on accuracy. Additionally, for both $6 \mathrm{Mbps}$ and $54 \mathrm{Mbps}$, HSSM is able to represent the measurement trends (adding the capture model increases the accuracy significantly). Furthermore, the impact from HSSM becomes more apparent for 54 Mbps. This is expected: as 54 Mbps is a less robust modulation coding rate, it benefits more from higher precision. The PHY layer results show that HSSM with capture is the most accurate model. We will next evaluate the impact of these models on transport layer protocols.

\section{Does Detail Matter to Upper Layers?}

The goal of our evaluation in this section is to understand the impact from more precise PHY-layer models on transport-layer performance in wireless simulators. Again, in all our experiments, we used either the lowest transmission rate $(6 \mathrm{Mbps})$ or the highest transmission rate (54 Mbps). The transport protocol was either UDP or TCP. We compare the testbed measurement results with four simulation models: (1) Threelogdist (2) BIM FER (Network-based), (3) BIM FER (Receiver-based) and (4) BIM FER (Link-based). We used nonlinear least squares (NLS) to find an appropriate fitting for each parameter of Threelogdist based on the measurement data. In the remainder of this section, we present our evaluations based on: (1) single flows and (2) multiple concurrent flows (see Table I).

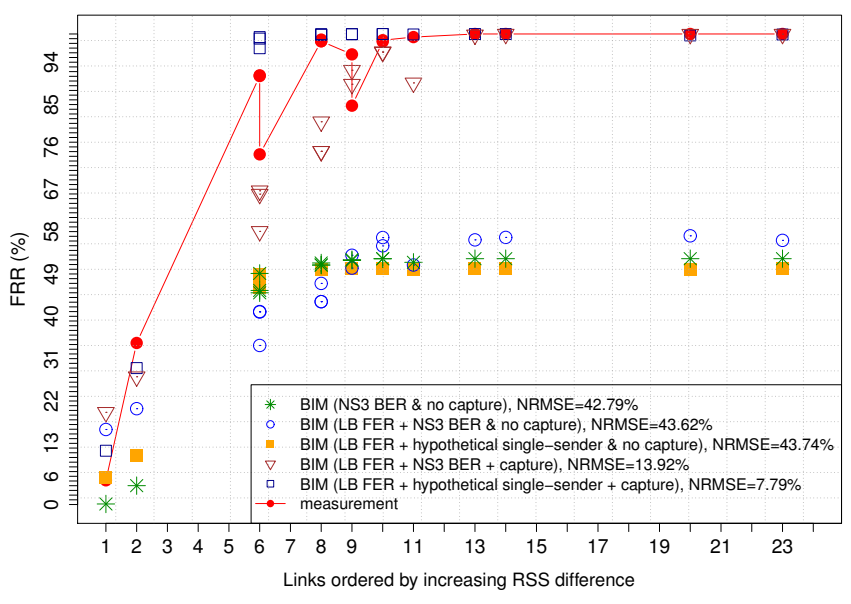

Fig. 6. Interference validation for $6 \mathrm{Mbps}$

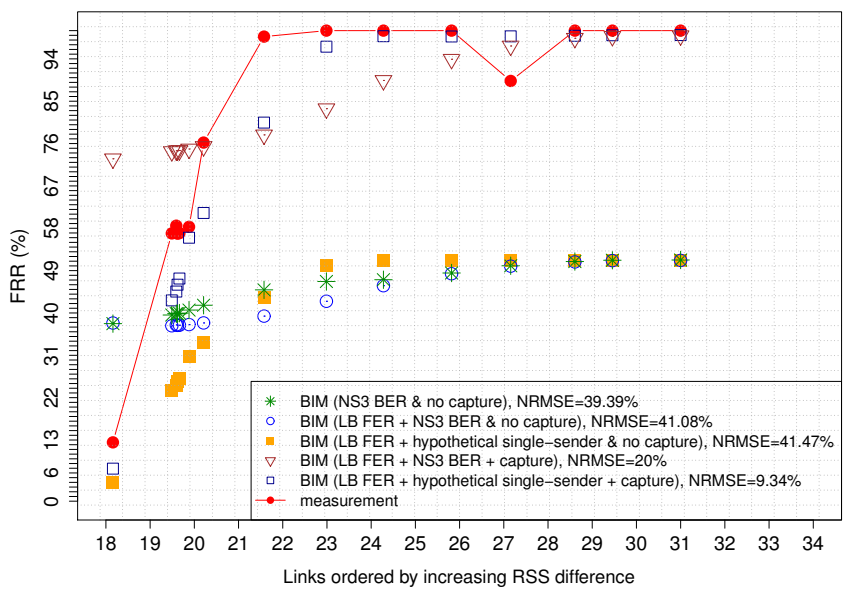

Fig. 7. Interference validation for $54 \mathrm{Mbps}$

\section{A. Single Flows}

Fig. 8(a) and Fig. 8(b) show the performance at $6 \mathrm{Mbps}$ with single flows when the transport protocol is UDP and TCP, respectively. We observe that indeed our site-specific model, BIM is able to capture the upper layer performance significantly better than both Friis and Threelogdist models. This underlines the importance of measurement-based models to represent the performance of a given network in simulations. Essentially, both Friis and Threelogdist models predict high throughput for the links that no packets could be delivered during measurements. BIM is able to represent such links better. For the gray area links, however, the situation is different. Even though, in Section IV-B, we obtain better frame reception ratio with more precise FER models, for transportlayer throughput, this does not play a role and the performance with BIM + NS3 BER model is comparable to FER models. Note that as TCP involves complex retransmission and timeout mechanisms, the NRMSE of all the evaluated models increase compared to the UDP case. We obtain similar trends when 


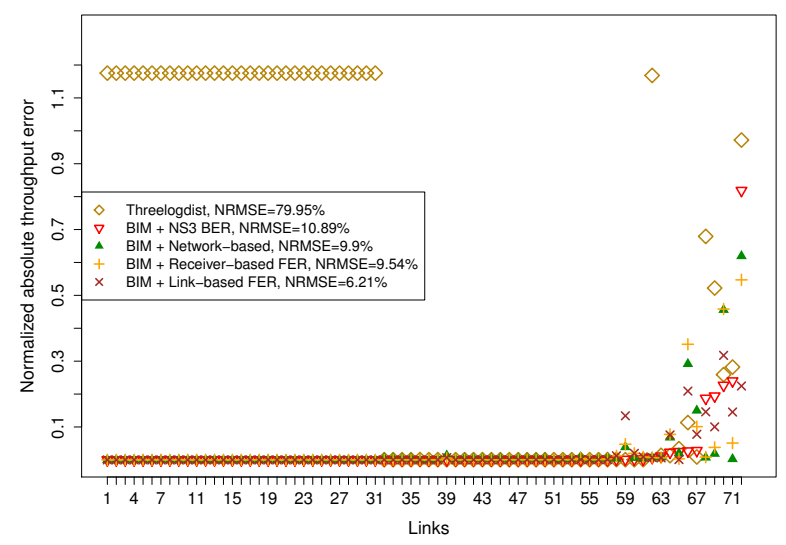

(a) UDP

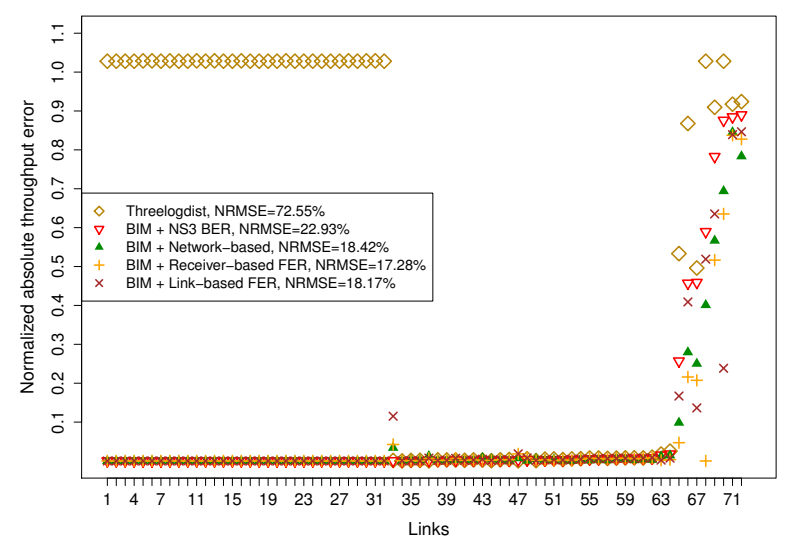

(b) TCP

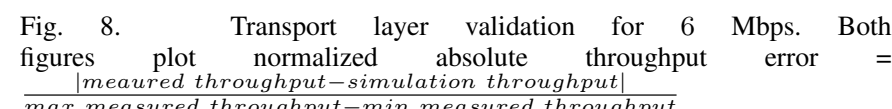

TABLE II

NRMSE FOR 54 MBPS FOR UDP AND TCP TRANSPORT PROTOCOLS.

\begin{tabular}{|l|l|l|}
\hline Model & UDP & TCP \\
\hline \hline Threelogdist & $32.15 \%$ & $42.84 \%$ \\
\hline BIM + NS3 BER & $6.05 \%$ & $2.41 \%$ \\
\hline BIM + Network-based FER & $7.76 \%$ & $5.9 \%$ \\
\hline BIM + Receiver-based FER & $9.35 \%$ & $7.43 \%$ \\
\hline BIM + Link-based FER & $8.75 \%$ & $6.07 \%$ \\
\hline
\end{tabular}

54 Mbps transmit rate is used (see Table II). Again Friis and Threelogdist models result in high NRMSE values, whereas the BIM model achieves significantly close performance to the measurement results. Also combining the observations with $6 \mathrm{Mbps}$ results, it is not possible to identify the best error model as all models perform comparably well and no model consistently outperforms the other.

\section{B. Multiple Concurrent Flows}

To evaluate the simulation models for the multiple concurrent flows scenario, we chose 25 different sets of six nodes. The communication graph of links in BOWL were used to
TABLE III

NRMSE OF THROUGHPUT, LIR AND JAIN'S FAIRNESS INDEX FOR 6 MBPS FOR BOTH UDP AND TCP. (THE RESULTS ARE IN \%.)

\begin{tabular}{|l||l|l|l|l||l|l|}
\hline \multirow{2}{*}{ Model } & \multicolumn{2}{l|}{ Throughput } & \multicolumn{2}{l|}{ LIR } & \multicolumn{2}{l|}{ Fairness } \\
\cline { 2 - 7 } & TCP & UDP & TCP & UDP & TCP & UDP \\
\hline \hline NS3 BER & 24.93 & 23.15 & 16.32 & 16.17 & 18.62 & 13.41 \\
\hline LB FER & 17.97 & 13.37 & 11.6 & 10.06 & 21.12 & 15.76 \\
\hline $\begin{array}{l}\text { LB FER } \\
+ \text { HSSM }\end{array}$ & 18.08 & 13.51 & 11.66 & 10.11 & 21.63 & 16.36 \\
\hline $\begin{array}{l}\text { LB FER } \\
\text { + Cap. }\end{array}$ & 15.55 & 17.45 & 10.8 & 11.73 & 16.3 & 11.09 \\
\hline $\begin{array}{l}\text { LB FER + } \\
\text { HSSM + Cap. }\end{array}$ & 18.24 & 20.64 & 13.26 & 15.25 & 13.72 & 10.62 \\
\hline
\end{tabular}

pick the participant nodes in the experiment. To create a set of six nodes, each time, a link was randomly chosen, and removed from the graph until three links are chosen. In this way, more than 100 three-link sets were found, out of which 25 are chosen. In each set, three nodes acted as senders and the remaining three acted as corresponding receivers. Each sender communicated with only a particular receiver and transmitted data with $5 M b p s$ for $120 \mathrm{~s}$ creating either TCP or UDP traffic.

We use three metrics to compare the accuracy of the different simulation models (the same models presented in Section IV-C): Throughput, Link Interference Ratio (LIR) [33] and Jain's fairness index. LIR is calculated as follows:

$$
\operatorname{LIR}_{A B, C D}=\frac{U_{A B}^{A B, C D}+U_{C D}^{A B C D}}{U_{A B}+U_{C D}},
$$

where $U_{A B}^{A B, C D}$ and $U_{C D}^{A B, C D}$ the unicast throughput for links $A B$ and $C D$ when they are active simultaneously, $U_{A B}$ and $U_{C D}$ are the throughput for links $A B$ and $C D$ when the link is the only link active in the environment.

Jain's fairness index is calculated as:

$$
J\left(x_{1}, x_{2}, \ldots, x_{n}\right)=\frac{\left(\sum_{i=1}^{n} x_{i}\right)^{2}}{n \cdot \sum_{i=1}^{n} x_{i}^{2}},
$$

where $x_{i}$ is the throughput for a flow $i$.

Fig. 9(a) shows the throughput results at 6 Mbps PHY layer transmit rate for TCP, whereas Fig. 9(b) shows LIR performance. For the sake of clarity of the figures, we depict three models: BIM (NS3 BER \& no capture), BIM (LB FER, HSSM and capture), BIM (LB FER, capture). The results for the rest of the models are summarized in Table III. Similar to the PHY layer results presented in Section IV-C, we see that the inclusion of the capture model improves simulation accuracy significantly.

\section{CONCLUSION}

In this paper, we have used extensive measurements to evaluate different simulation models, which have different levels of precision. In particular, we investigated the importance of detailed frame error rate (FER), and interference and capture models. Our study allows us to understand the level of detail needed in wireless simulations to be able to represent the real network behavior. For instance, while more accuracy at the PHY layer can typically be obtained with more precise models, this is not always the case for higher layers (transport layer in 


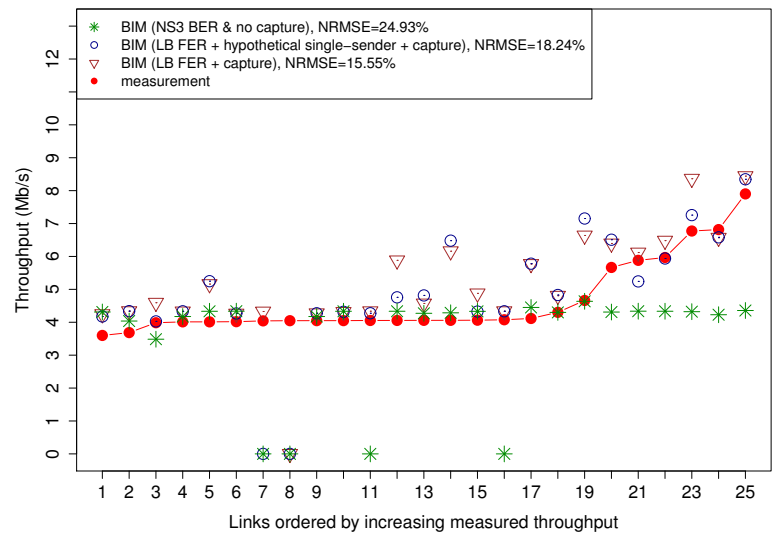

(a) Throughput

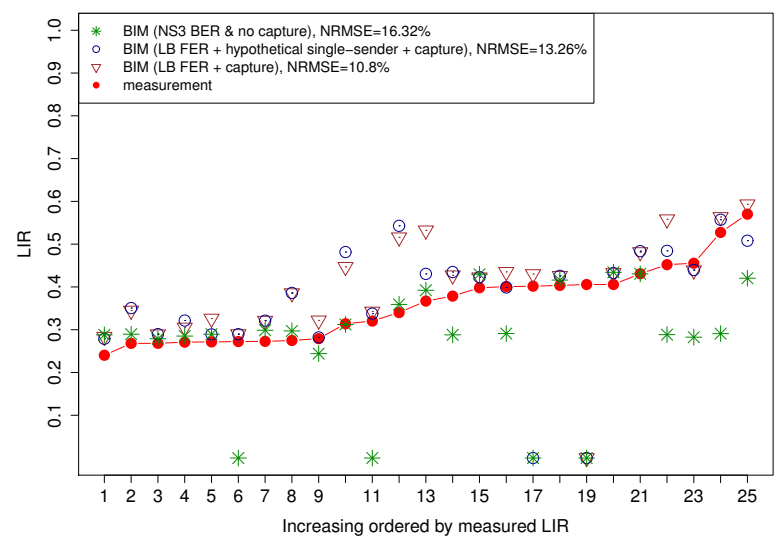

(b) LIR

Fig. 9. Transport layer validation (multi-flow) with TCP for 6 Mbps.

this paper). Even at the PHY layer, we have shown that precise models only help with a small percentage of more dynamic links, while "strong" and "weak" links can afford simpler models. Our results underline the importance of modeling the behavior of wireless network cards (e.g., Atheros MIM capture behavior), which especially play the most significant role for concurrent multiple flows. For future work, we plan to investigate the accuracy of BIM models with higher number of single-hop and multi-hop flows, and different traffic models.

\section{REFERENCES}

[1] S. Kurkowski, T. Camp, and M. Colagrosso, "Manet simulation studies: The incredibles," SIGMOBILE Mob. Comput. Commun. Rev., vol. 9, pp. $50-61,2005$

[2] N. Baldo and M. Miozzo, "Spectrum-aware channel and PHY layer modeling for ns3," in Intl. Workshop on Network Simulation Tools (NSTOOLS), Oct. 2009.

[3] A. Kashyap, S. Ganguly, and S. R. Das, "Measurement-based approaches for accurate simulation of 802.11-based wireless networks," in MSWIM, 2008, pp. 54-59.

[4] S. Papanastasiou, J. Mittag, E. G. Strom, and H. Hartenstein, "Bridging the gap between physical layer emulation and network simulation," in IEEE WCNC, Apr. 2010, pp. 1-6.

[5] D. Aguayo, J. Bicket, S. Biswas, G. Judd, and R. Morris, "Link-level measurements from an $802.11 \mathrm{~b}$ mesh network," SIGCOMM Comput. Commun. Rev., vol. 34, no. 4, pp. 121-132, 2004.
[6] J. Bicket, D. Aguayo, S. Biswas, and R. Morris, "Architecture and evaluation of an unplanned 802.11b mesh network," in MobiCom, 2005 , pp. 31-42.

[7] S. Ivanov, A. Herms, and G. Lukas, "Experimental validation of the ns-2 wireless model using simulation, emulation, and real network," in WMAN, 2007, pp. 433-444.

[8] M. Al-Bado, R. Merz, C. Sengul, and A. Feldmann, "A site-specific indoor link model for realistic wireless network simulations," in 4th International ICST Conference on Simulation Tools and Techniques (SIMUTOOLS), March 2011

[9] R. Merz, H. Schiöberg, and C. Sengul, "Design of a configurable wireless network testbed with live traffic," in TridentCom, May 2010.

[10] "ns-3." [Online]. Available: \url\{http://www.nsnam.org/\}

[11] "ns-2." [Online]. Available: \url\{http://www.isi.edu/nsnam/ns/ ns-documentation.html

[12] "Opnet," http://www.opnet.com/.

[13] "Qualnet," http://www.scalable-networks.com/products/qualnet/.

[14] I. Gruber, O. Knauf, and H. Li, "Performance of ad hoc routing protocols in urban environments," in European Wireless, 2004.

[15] C. Newport, D. Kotz, Y. Yuan, R. S. Gray, J. Liu, and C. Elliott, "Experimental evaluation of wireless simulation assumptions," SIMULATION, vol. 83, no. 9, pp. 643-661, Sep. 2007.

[16] T. Rappaport, Wireless Communications: Principles and Practice. Upper Saddle River, NJ, USA: Prentice Hall PTR, 2001.

[17] A. Iyer, C. Rosenberg, and A. Karnik, "What is the right model for wireless channel interference?" IEEE Transactions on Wireless Communications, vol. 8, no. 5, pp. 2662-2671, May 2009.

[18] N. Baldo, F. Maguolo, M. Miozzo, M. Rossi, and M. Zorzi, "ns2miracle: a modular framework for multi-technology and cross-layer support in network simulator 2," in 2nd Intl. conference on Performance evaluation methodologies and tools, ser. ValueTools '07, 2007, pp. 1-8.

[19] Q. Chen, F. Schmidt-Eisenlohr, D. Jiang, M. Torrent-Moreno, L. Delgrossi, and H. Hartenstein, "Overhaul of ieee 802.11 modeling and simulation in ns-2," in MSWiM '07, 2007, pp. 159-168.

[20] M. Lacage and T. R. Henderson, "Yet another network simulator," ser. WNS2 '06. ACM, 2006.

[21] "Ns-3 model library," http://www.nsnam.org/docs/release/3.11/models/ ns-3-model-library.pdf, May 2011.

[22] "Cost action 231," http://www.lx.it.pt/cost231/final_report.htm.

[23] W. C. Jakes and D. C. Cox, Eds., Microwave Mobile Communications. Wiley-IEEE Press, 1994.

[24] J. Ryu, J. Lee, S. Lee, and T. Kwon, "Revamping the IEEE 802.11a PHY simulation models," in MSWIM, 2008, pp. 28-36.

[25] J. Manweiler, N. Santhapuri, S. Sen, R. R. Choudhury, S. Nelakuditi, and K. Munagala, "Order matters: Transmission reordering in wireless networks," in MobiCom, Sep. 2009.

[26] J. Liu, Y. Yuan, D. M. Nicol, R. S. Gray, C. C. Newport, D. Kotz, and L. F. Perrone, "Empirical validation of wireless models in simulations of ad hoc routing protocols," Simulation, vol. 81, pp. 307-323, April 2005.

[27] P. J. Husted, Y. Huanchun, and S. Aman, "Adaptive interference immunity control," US Patent 7349503, Atheros Communications, Inc., March 2008.

[28] I. Tinnirello, D. Giustiniano, L. Scalia, and G. Bianchi, "On the sideeffects of proprietary solutions for fading and interference mitigation IEEE 802.11b/g outdoor links," Computer Networks, Feb. 2009.

[29] "Atheros," http://www.wifi-stock.com/file/cm9_user_manual.pdf.

[30] "Bowl indoor model in ns-3," http://code.nsnam.org/rmerz/ns-3-bimsimutools2011.

[31] "IEEE standard for information technology-telecommunications and information exchange between systems-local and metropolitan area networks-specific requirements - Part 11: wireless lan medium access control (MAC) and physical layer (PHY) specifications," pp. C1 -1184 June 2007.

[32] C. Reis, R. Mahajan, M. Rodrig, D. Wetherall, and J. Zahorjan, "Measurement-based models of delivery and interference in static wireless networks," in ACM SIGCOMM, 2006, pp. 51-62.

[33] J. Padhye, S. Agarwal, V. Padmanabhan, L. Qiu, A. Rao, and B. Zill, "Estimation of link interference in static multi-hop wireless networks," in $I M C$, Oct. 2005 